\title{
Quantifying fibre reorientation during axial compression of a composite through time-lapse $X$-ray imaging and individual fibre tracking
}

DOI:

10.1016/j.compscitech.2018.08.028

\section{Document Version}

Accepted author manuscript

Link to publication record in Manchester Research Explorer

Citation for published version (APA):

Emerson, M. J., Wang, Y., Withers, P. J., Conradsen, K., Dahl, A. B., \& Dahl, V. A. (2018). Quantifying fibre reorientation during axial compression of a composite through time-lapse $X$-ray imaging and individual fibre tracking. Composites Science and Technology, 168, 47-54. https://doi.org/10.1016/j.compscitech.2018.08.028

Published in:

Composites Science and Technology

\section{Citing this paper}

Please note that where the full-text provided on Manchester Research Explorer is the Author Accepted Manuscript or Proof version this may differ from the final Published version. If citing, it is advised that you check and use the publisher's definitive version.

\section{General rights}

Copyright and moral rights for the publications made accessible in the Research Explorer are retained by the authors and/or other copyright owners and it is a condition of accessing publications that users recognise and abide by the legal requirements associated with these rights.

\section{Takedown policy}

If you believe that this document breaches copyright please refer to the University of Manchester's Takedown Procedures [http://man.ac.uk/04Y6Bo] or contact uml.scholarlycommunications@manchester.ac.uk providing relevant details, so we can investigate your claim.

\section{OPEN ACCESS}




\title{
Quantifying Fibre Reorientation during Axial Compression of a Composite through Time-lapse X-ray Imaging and Individual Fibre Tracking
}

\author{
Monica Jane Emerson ${ }^{\mathrm{a}, *}$, Ying Wang ${ }^{\mathrm{b}}$, Philip John Withers ${ }^{\mathrm{b}}$, Knut \\ Conradsen $^{\mathrm{a}}$, Anders Bjorholm Dahl ${ }^{\mathrm{a}}$, Vedrana Andersen Dahl ${ }^{\mathrm{a}}$ \\ ${ }^{a}$ Section for Image Analysis and Computer Graphics, Department of Applied Mathematics \\ and Computer Science, Technical University of Denmark, Kongens Lyngby, 2800, Denmark. \\ ${ }^{b}$ Henry Moseley X-ray Imaging Facility, School of Materials, The University of Manchester, \\ Manchester, M13 9PL, United Kingdom.
}

\begin{abstract}
The sudden compressive failure of unidirectional (UD) fibre reinforced composites at loads well below their tensile strengths is a cause of practical concern. In this respect and more generally, analytical and numerical models that describe composite behaviour have been hard to verify due to a lack of experimental observation, particularly in 3D. The aim of this paper is to combine fast in-situ $\mathrm{X}$-ray computed tomography $(\mathrm{CT})$ with advanced image analysis to capture the changes in fibre orientation in 3D during uninterrupted progressive loading in compression of a UD glass fibre reinforced polymer (GFRP). By analysing and establishing correspondence between a sequence of time-lapse X-ray CT images of the composite, we are able for the first time to follow each fibre and quantify the progressive deflection that takes place during axial compression in the steps leading up to fibre micro-buckling and kinking. Even at just $25 \%$ of the failure load, fibres have started to tilt in approximately the direction of the ultimate kink band. The rate of tilting increases as the composite approaches the collapse load. More generally, our approach can be applied to investigate the behaviour of a wide range of fibrous materials under changing loading conditions.
\end{abstract}

Keywords: A. Carbon fibres; B. Mechanical properties; C. Damage

\footnotetext{
${ }^{*}$ Corresponding author

Email address: monj@dtu.dk (Monica Jane Emerson)
}

Preprint submitted to Composites Science and Technology

October 26, 2018 
mechanisms; C. Buckling; Fibre misalignment

\section{Introduction}

Composite materials are used extensively in advanced structures where properties such as high stiffness and low weight are required. In particular, carbon and glass fibre reinforced polymers (CFRP and GFRP) are being employed in5 creasingly in aero [1] and ground transportation, as well as in environmentally sustainable energy production systems, such as wind turbines 2 . Therefore, it is crucial to understand their performance under realistic loading conditions with the ultimate goal of optimising performance through the use of analytical or numerical models [3]. Currently, the lack of confidence in these models means that expensive experimental mechanical testing is relied upon in the development of new composite structures, and is still the gold standard for quality control in industry [4]. What is more, this lack of confidence in the prediction of composite behaviour, together with high safety requirements, leads to an unnecessary over-engineering of the components.

The compressive strengths of unidirectional (UD) composites are typically significantly below their tensile strengths [5]. This is because many of the mechanisms of compressive failure are dictated largely by the matrix properties, in contrast to the tensile properties which are dictated by the fibres. A number of failure mechanisms under compression have been described in $[6] 8$, the most important of which for the current study is fibre micro-buckling (see Fig. 1. (bc)). Longitudinal splitting along the fibre direction is also a potential damage mode (see Fig. 1(d)). Fibre micro-buckling is highly sensitive to the initial fibre misalignment [6, 9]. As a result of the composite manufacturing process, the fibres are, in general, not perfectly aligned with the axial direction. This means that when compression is applied, some level of shear is induced in the region of the misaligned fibres. When the local shear stress exceeds the matrix shear yield strength, the matrix yields and fibres buckle. In other words, the compression strength of a UD composite relies on the ability of the matrix material to 
keep the fibres straight [5]. Fibres finally fracture when the strain on the compressive/tensile side of the fibre exceeds the fibre's compressive/tensile failure strain, and a kink band is formed [10. Although for the reasons discussed above many researchers believe that fibre misalignment plays a big role [11, 12, and the degree of lateral constraint on the fibres from the matrix is important [13], the initiation mechanism for fibre micro-buckling is still a subject of debate.

35 This is largely because of the lack of direct evidence of the fibre movements in the moments leading up to micro-buckling and the point at which this becomes a kink band. The difficulty in capturing experimentally the precursors to failure is partly due to the small levels of misalignment required to trigger instability, and partly because of the sudden and catastrophic nature of the instability when 40 the kink bands form.

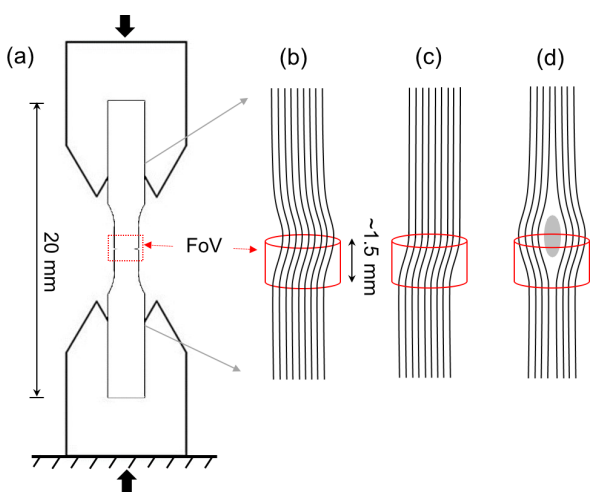

Figure 1: Schematic illustration of a loaded specimen and damage modes associated with axial compression. (a) Composite specimen geometry and loading condition, and potential damage modes associated with axial compression failure of unidirectional fibre composite, namely (b-c) fibre micro-buckling and (d) longitudinal splitting.

In recent years, optical and scanning electron microscopy have increased in image acquisition speeds. This has been exploited to study the development of kink bands in composites loaded in-situ [14 by observing the surface of the composites. Guynn et al. [14 and more recently Moran et al. 15] have observed experimentally in 2D that fibres buckle before a kink band is formed. However, the stage of fibre deflection prior to the onset of kink-band formation, which is 
expected to influence the kink-band geometry, has not yet been captured experimentally. This is partly because $2 \mathrm{D}$ characterisation techniques cannot capture information out of the observed plane. X-ray computed tomography (CT) enables 3D imaging of FRP specimens containing many fibres at a resolution where individual fibres can be resolved [16]. Wang et al. [17] employed post-mortem X-ray CT to study kink-band formation and reported the 3D morphology of kink bands for the first time, where deformation out of the kinking plane was also observed within the damage zone. Moreover, it is possible to scan a sample while it is loaded in-situ in compression so that the evolution in fibre orientation under load can be observed in 3D from no load towards failure. Conventional tomography, even at synchrotron sources, is too slow to capture the onset of micro-buckling [18, 19]. However, ultra-fast imaging $(\sim 500-10,0002 \mathrm{D}$ images per second) is now possible on some beamlines, which enables the events leading up to failure to be captured by X-ray CT 20. Using ultra-fast in-situ imaging, Wang 21 obtained a time-series of 3D X-ray CT images leading up to kink bands, extending on the work [17; while the quantification of the small preceding fibre reorientation requires advanced image analysis.

The focus of this paper is on combining in-situ X-ray CT with advanced image analysis with the purpose of providing an experimental description of the changes in fibre orientation during progressive loading in compression. Fibre orientations have been estimated from tomograms [22 24] without previously segmenting individual fibres, but the accuracy of these estimates is highly dependent on the quality of the tomograms. However, if individual fibres can be segmented beforehand, the accuracy of the individual fibre trajectories can be ascertained prior to computing fibre orientations. Additionally, segmentation of individual fibres opens up the possibility of following the changes in trajectory for each individual fibre across successive loading steps. Methods that can segment individual fibres from tomograms of UD FRPs, where fibres are closely 75 packed, have been applied to measure fibre geometry from high-quality data 25 and also from lower quality scans $26-28$. The method by Czabaj et al. [26] involves a time-consuming validation of the determined fibre trajectories and 
is therefore applicable to small volumes containing a limited amount of fibres. Sencu et al. 27] built a micro-mechanical model with a few fibres that had been segmented from bundles orientated in different directions. The focus of Emerson et al. 28] was on determining individual fibre orientations. The method by Emerson et al. ensures a representative characterisation of the composite's microstructure due to its ability to find a large amount of fibres and delineate their centre line with high precision, even at resolutions where a fibre diameter 85 is covered by just 4 pixels 29 .

Our proposed methodology is to characterise the trajectory of individual fibres in the as-manufactured condition in 3D from X-ray CT images and then to quantify, as compressive loading progresses, the deflections by the analysis of a time-lapse CT image sequence. The aim is to capture precursors to fibre micro-buckling and kinking in 3D for the first time, in the moments leading up to kink-band formation. The material system employed in the experiment was an end-tabbed UD GFRP rod specimen (see Fig. 11(a)).

\section{Materials and experimental procedures}

\subsection{Manufacturing and preparation of composite sample}

A modified resin infusion technique referred to as small-scale resin infusion, as reported by Wang et al. [17, was used to fabricate the $2 \mathrm{~mm}$ diameter UD E-glass fibre (12 $\mathrm{mm}$ in diameter)/epoxy (Huntsman Araldite LY564/XB3486) composite rods. Specimens with a reduced gauge section of around $1.5 \mathrm{~mm}$ in diameter over $3 \mathrm{~mm}$ in length were prepared from the manufactured composite rods. Fig. 1(a) shows the specimen geometry for in-situ testing. So as to localise the damage site, a groove around the circumference was made using a razor blade giving a notch depth between $100 \mu \mathrm{m}$ and $200 \mu \mathrm{m}$. The two ends of the specimen were then glued with an epoxy adhesive into chamfered steel end caps so that end-splitting damage could be avoided. This resulted in a length of the composite rod equal to $20 \mathrm{~mm}$ and an overall length of the specimen of $\sim 28 \mathrm{~mm}$. 


\subsection{Ultra-fast synchrotron X-ray computed tomography (CT) imaging under}

in-situ compression

Ultra-fast imaging was performed at the TOMCAT beamline from the Swiss

\section{Methods}

To follow the evolution of individual fibres from tomogram to tomogram as the load is increased, we employ the segmentation method by Emerson et al. 28] for its ability to segment large fields of view and its robustness to im- 


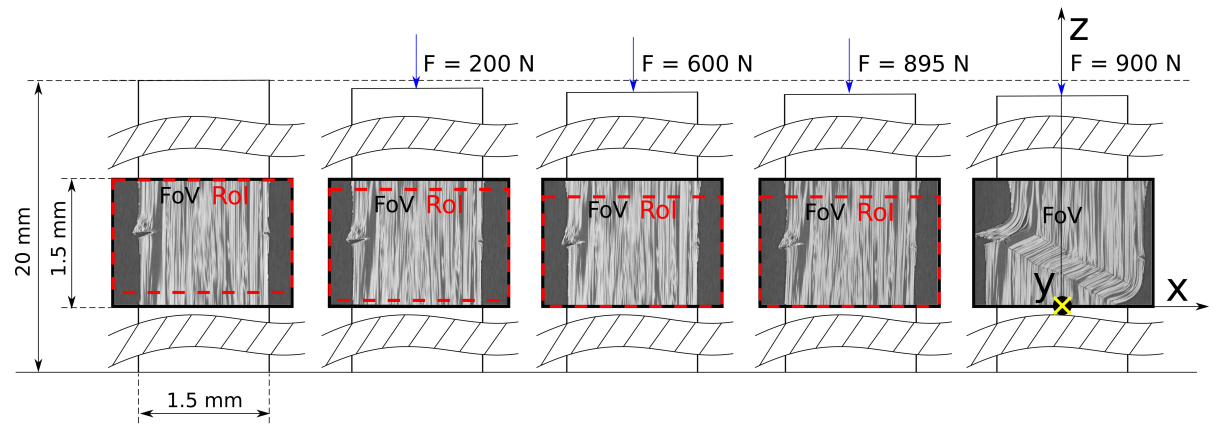

Figure 2: X-ray CT longitudinal slices from the centre of the composite rod in the direction of the kink-band plane. The composite rod of length $20 \mathrm{~mm}$ and of diameter $1.5 \mathrm{~mm}$ at the reduced gauge section was scanned with a field of view (FoV) of $1.5 \mathrm{~mm}$ in height during uninterrupted in-situ axial compression loading from $0 \mathrm{~N}$ to $900 \mathrm{~N}$. Images corresponding to four loads are selected for the analysis: i) $0 \mathrm{~N}$, ii) $200 \mathrm{~N}$, iii) $600 \mathrm{~N}$ and iv) $895 \mathrm{~N}$; and the analysed region of interest (RoI) has a height of approximately $1.4 \mathrm{~mm}$. The coordinate system is defined according to the loading direction ( $z$-axis, inclination $\phi=0)$ and the kink-band plane $(x$-axis, azimuth $\theta=0)$.

correspondence is established between the individual fibres across data-sets.

Once individual fibre trajectories are obtained and matched across the four data-sets, individual fibre orientations are computed at every load step, and individual fibre deflections are calculated as the change in fibre orientation with respect to the as-manufactured fibre orientation.

\subsection{Determination of individual fibre trajectories}

The UD fibres are essentially aligned with the $z$-axis, so in each horizontal - also called cross-sectional - slice through the volume fibres appear as circles, as shown in Fig. 3. This allows the detection of fibre centres in each crosssectional slice, after which the detections are connected through the volume to form individual fibres. Each individual fibre centre line is called a 'trajectory', which is the curve that connects the centres of a fibre through the volume. These trajectories are extracted in two steps: i) centre coordinates are detected for every fibre in each 2D tomographic cross-sectional slice, obtained by slicing the $3 \mathrm{D}$ volume in the direction orthogonal to the fibres, and ii) 3D fibre trajectories 
are determined by tracking, i.e. connecting the $2 \mathrm{D}$ detected centres that belong to the same fibre.

The process of centre coordinate detection is illustrated and explained in Fig. 3. First, a dictionary-based probabilistic segmentation is applied so as to estimate pixel-wise probabilities of belonging to the centre region. Then, the resulting probability map is thresholded to obtain the individual centre regions. Finally, centre coordinates are computed as the centres of mass of the connected regions. For more details refer to Emerson et al. [28].

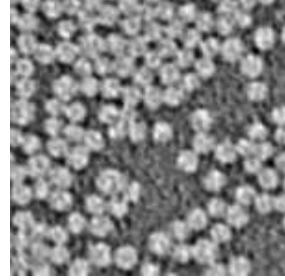

(a) Intensity image

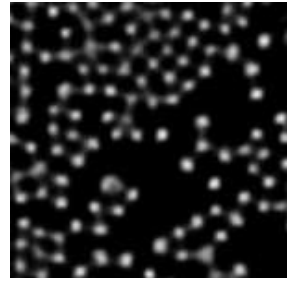

(b) Probability map

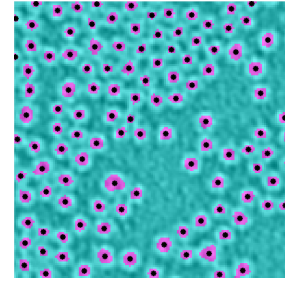

(c) Segmentation

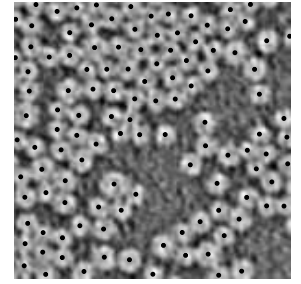

(d) Detected fibre centres

Figure 3: The steps in detecting fibre centres from 2D cross-sectional slices. The intensity image in (a) is segmented into two classes (fibre centre regions or not). The probability map in (b) is obtained using the dictionary-based probabilistic segmentation method in 28, and indicates the likelihood of each pixel belonging to the fibre centre class. The probability map is thresholded to obtain the segmentation in (c), which determines what are fibre centre regions (pink) or not (blue). The centre coordinates for the individual fibres, marked in black over (c-d), are found as the centroids of the pink connected regions.

A new implementation of the dictionary-based segmentation method is available 32. The main improvements are lower computational time for training the dictionary model as well as for the segmentation phase and, most importantly, the development of a graphical user interface (GUI) in Matlab ${ }^{\circledR}$. The GUI wraps up the algorithm and provides a user-friendly interactive segmentation tool. The segmentation algorithm behind the GUI requires minimal user input for training the dictionary and, thanks to the interactive nature of the GUI, the amount of input is adaptable to the complexity and quality of the data-set at hand.

The process of fibre tracking for connecting the detected centres across the 
2D slices is explained in Emerson et al. 28. The fibre tracking used here is an the RoI and in the last slice of the RoI, while the intermediate slices are used only for tracking individual fibres.

version. This includes handling of i) double detections, by merging $2 \mathrm{D}$ fibre centre detections when these are closer than the manufacturer specified fibre radius, and handling of ii) merging fibres, by matching fibre centres from one slice to the next only if the match between the points is bidirectional, as opposed to utilising the unidirectional backward tracking approach in Emerson et al. 28.

\subsection{Matching corresponding fibre trajectories across loading steps}

As mentioned in Section 2.2, there is an angular disregistry between each data-set in the $x y$ plane and this needs to be corrected so that individual fibres can be followed across the data-sets. Corresponding fibre trajectories are matched across the four microtomograms using a rigid registration for alignment, followed by a nearest neighbour search algorithm for matching corresponding fibres.

Tomograms are aligned manually, first in the $z$ direction by visual inspection and then in the horizontal direction by choosing three corresponding points and calculating a rigid transformation (rotation plus translation). The corresponding points are selected as far as possible from each other and towards the edges of the cross-sectional slice to ensure precision in the registration.

\subsection{Definition of the vectors inside the deflection model}

Fig. 4 illustrates the evolution of a few randomly selected fibre trajectories as the composite is loaded in compression from $0 \mathrm{~N}$ to $895 \mathrm{~N}$. In essence, each slightly curved fibre trajectory can be approximated by a sequence of straight line segments computed for different horizontal segments. Since the curvature of the fibres is small within the RoI, for our current analysis we approximate each fibre by a single straight line through the RoI. This approximation implies that our analysis is based on the position of the fibre centres in the first slice of 


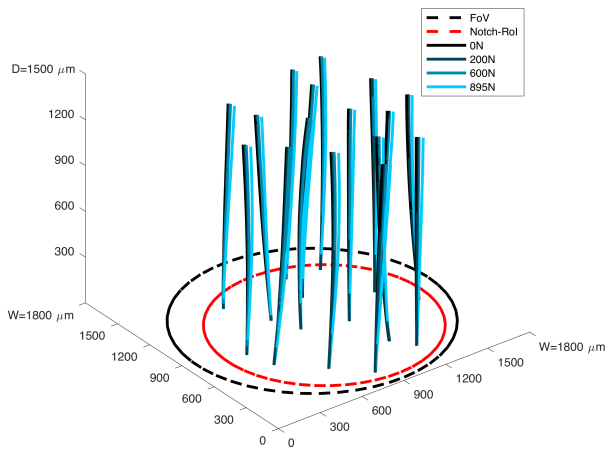

(a)

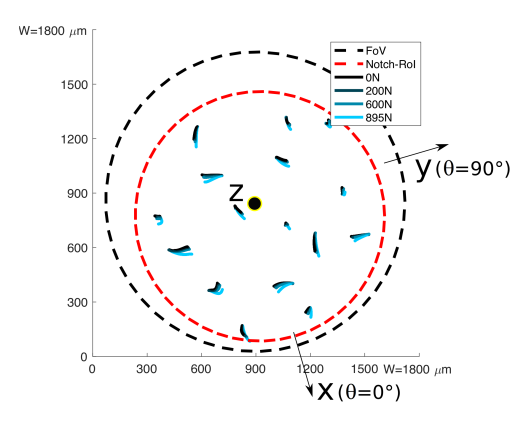

(b)

Figure 4: The evolution of the fibre trajectories under load. In (a) a 3D side view and in (b) a plan view showing the evolution under compression of randomly selected fibres inside the RoI. The coordinate system, defined according to the loading direction and the kink-band plane, is indicated in (b).

We quantify the fibre trajectories and deflections during loading by analysing the straight line segments that approximate the fibres. We have a time series of four line segments per fibre, one for each loading step. For the sake of analysis, we define a set of vectors to describe the orientation and deflection of both the individual fibres and the composite as a whole. These vectors are described pictorially in Fig. 5 .

The fibre trajectory for the $n$-th fibre under the $i$-th load state is captured by the fibre vector $\mathbf{v}_{\mathbf{f}_{n}}^{i}$. This vector joins the points where the fibre $\mathrm{f}_{n}$ enters and leaves the RoI, and is defined for $\mathrm{F}_{i} \in\{0 \mathrm{~N}, 200 \mathrm{~N}, 600 \mathrm{~N}, 895 \mathrm{~N}\}$ and $n \in$ $\{1, \ldots, N\}$, with $N=4957$ the number of fibres considered in this study. The corresponding vector for the composite is the average of all the fibre vectors and is denoted by the composite vector $\mathbf{v}_{\mathrm{c}}^{i}=\frac{1}{N} \sum_{n=1}^{N} \mathbf{v}_{\mathrm{f}_{n}}^{i}$.

${ }_{210}$ The fibre deflection for the $n$-th fibre at the $i$-th load state considers only the lateral deflection of the fibre end relative to its orientation in the $0 \mathrm{~N}$ load case, and is represented by the fibre deflection vector $\mathbf{d}_{\mathbf{f}_{n}}^{i}$. It is calculated for fibre $\mathrm{f}_{n}$ as the difference between the fibre vector for $\mathrm{f}_{n}$ at load step $i$ and the fibre vector for $\mathrm{f}_{n}$ at load $\mathrm{F}_{1}=0 \mathrm{~N}$, i.e. $\mathbf{d}_{\mathrm{f}_{n}}^{i}=\mathbf{v}_{\mathrm{f}_{n}}^{i}-\mathbf{v}_{\mathrm{f}_{n}}^{1}$. The composite 
by the mean of all the fibre deflection vectors, i.e. $\mathbf{d}_{\mathrm{c}}^{i}=\frac{1}{N} \sum_{n=1}^{N} \mathbf{d}_{\mathrm{f}_{n}}^{i}$. Notice that $\mathbf{d}_{\mathrm{c}}^{i}=\mathbf{v}_{\mathrm{c}}^{i}-\mathbf{v}_{\mathrm{c}}^{1}$ and that the deflection vectors at load $\mathrm{F}_{1}=0 \mathrm{~N}, \mathbf{d}^{1}$, are all zero.

Since fibres are not free to move independently of one another, an additional important composite movement is given by the twist, as observed by Ueda et al. 33. The twist is caused by the torque induced by the compression loading and involves the fibres rotating collectively around the central axis of the composite rod. To estimate the angle of the twist, we introduce the relative deflection, which expresses the movement of a fibre relative to the deflection of the composite rod as a whole. It is represented by the relative deflection vector $\mathbf{r}_{\mathrm{f}_{n}}^{i}=\mathbf{d}_{\mathrm{f}_{n}}^{i}-\mathbf{d}_{\mathrm{c}}^{i}$. The twist angle $\gamma_{\mathrm{c}}$ is estimated by fitting a rotation to the relative deflection vectors and minimising the residual in the least squares sense 34 .
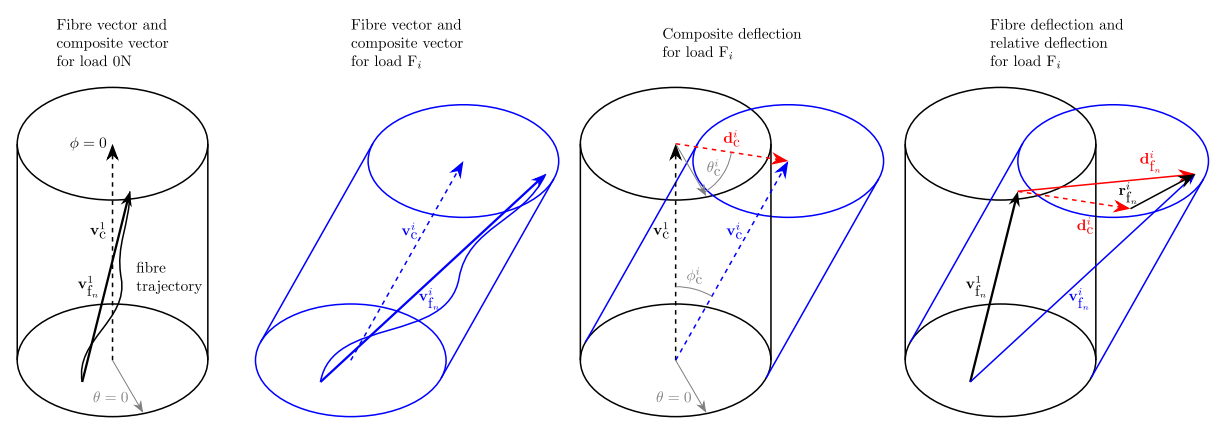

Figure 5: Definition of fibre and composite vectors, deflection vectors and relative deflection vectors.

When interpreting our results, the vectors are described using either Cartesian or spherical coordinates where i) the $z$-axis (inclination $\phi=0$ ) is aligned with the loading $z$-axis and points in the direction opposite to the loading direction, and ii) the $x$-axis (azimuth $\theta=0$ ) is aligned with the kink-band plane, pointing towards the shallowest part of the notch (for clarification see Figs. 2 and 5]. 


\section{Results and discussion}

\subsection{Characterisation of fibre orientations before loading}

It has been widely accepted that fibre misalignment in the as-manufactured composite can have a significant effect on the compressive failure load at which catastrophic kink bands form 6]. Fig. 6 shows the fibre trajectories in the as-manufactured data-set under $0 \mathrm{~N}$ load. It is evident from the figure that the fibres are predominantly straight over the $1.5 \mathrm{~mm}$ high $\mathrm{FoV}$, and that the orientation of the fibres is to a large extent grouped into clusters.

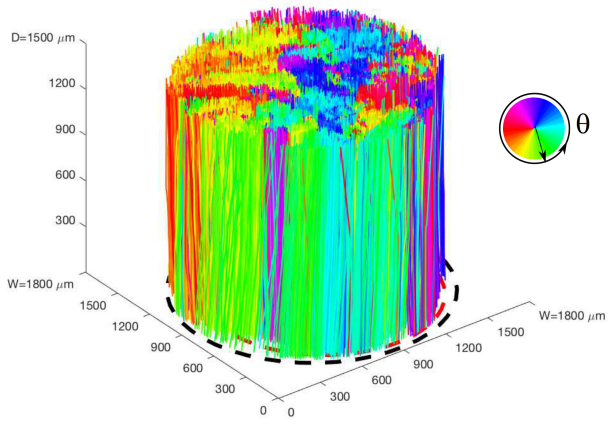

(a)

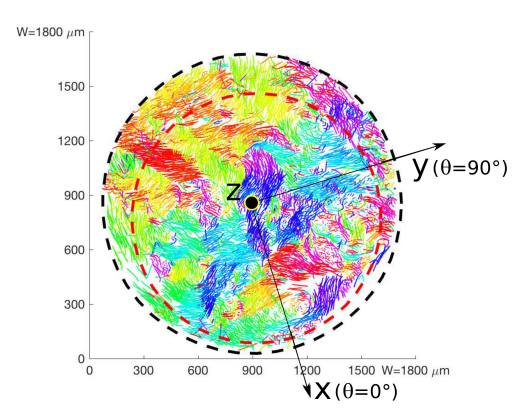

(b)

Figure 6: The as-manufactured fibre trajectories. In (a) a 3D side view and in (b) a plan view of all the fibre trajectories extracted from the RoI for the as-manufactured test piece prior to the application of load. The colour of each fibre encodes the azimuth $\theta$ of its fibre vector (defined in Fig. 5). The projected length in the plan view shows the amount of misalignment, fibres appear as points when they are completely aligned with the $z$-axis $(\phi=0)$. The coordinate system, defined according to the loading direction and the kink-band plane, is indicated in (b).

\subsection{Characterisation of fibre orientations during loading}

The spatial clustering of fibre orientations as a function of loading is shown in Fig. 7. The scatter plots (top) show the end points of the individual fibre vectors $\mathbf{v}_{\mathrm{f}_{n}}^{i}$ projected onto the horizontal plane. The colour-coding reflects the inclination and the azimuth of each fibre vector, such that saturation is low for 
fibres aligned with $z$. The hue indicates the azimuth of the fibre vector, and thus represents the in-plane misorientation direction of a fibre. Had all fibres been perfectly aligned, all points would be on top of each other and all colours the same. When looking at the scatter plots one might underestimate the number of highly aligned fibres, as the scatter plots do not show the density of points when these are closely clustered together. That is, there are many more aligned fibres than it seems when looking at the scatter plots.

With regards to the fibre orientations displayed in Fig. 7, it can be concluded that both the degree of fibre misalignment relative to the loading axis $\phi$ (fibre tilt) and the direction of the misalignment $\theta$ (fibre direction) cluster spatially. It is noteworthy that the clustering of the fibre direction is correlated with the local fibre volume fraction. Where fibres are very close together they tend to be orientated in the same direction whereas there tends to be a resin-rich region in between the differently orientated clusters of fibres. This is a reflection of the packing efficiency which drops when fibres are not similarly aligned. It is notable that while there is a gradual drift of the composite (as indicated by the black crosses over the scatter plots in Fig. 7) there are no significant changes in the clustering of fibre misalignments as the load is increased. This confirms that the fibres deform in a cooperative way in FRPs with relatively high fibre volume fraction. As to the evolution of the fibre vectors under progressive loading, a gradual deflection in the direction of the ultimate kink-band plane is observed in the scatter plots as the loading increases. This shift translates to an increase of green colour in the cross-sectional images under increasing load.

\subsection{Evolution of fibre deflections under load}

The spatial distribution of the deflection introduced by loading the sample is analysed by looking at the deflection vectors and the relative deflection vectors.

As was mentioned previously, the fibre deflection vectors $\mathbf{d}_{\mathbf{f}_{n}}^{i}$ capture the change in orientation that each individual fibre undergoes with respect to the $0 \mathrm{~N}$ state as a consequence of applying a certain load $\mathrm{F}_{i}$. The deflection vectors are displayed in the scatter plots of Fig. 8 - we omit the illustration of the 

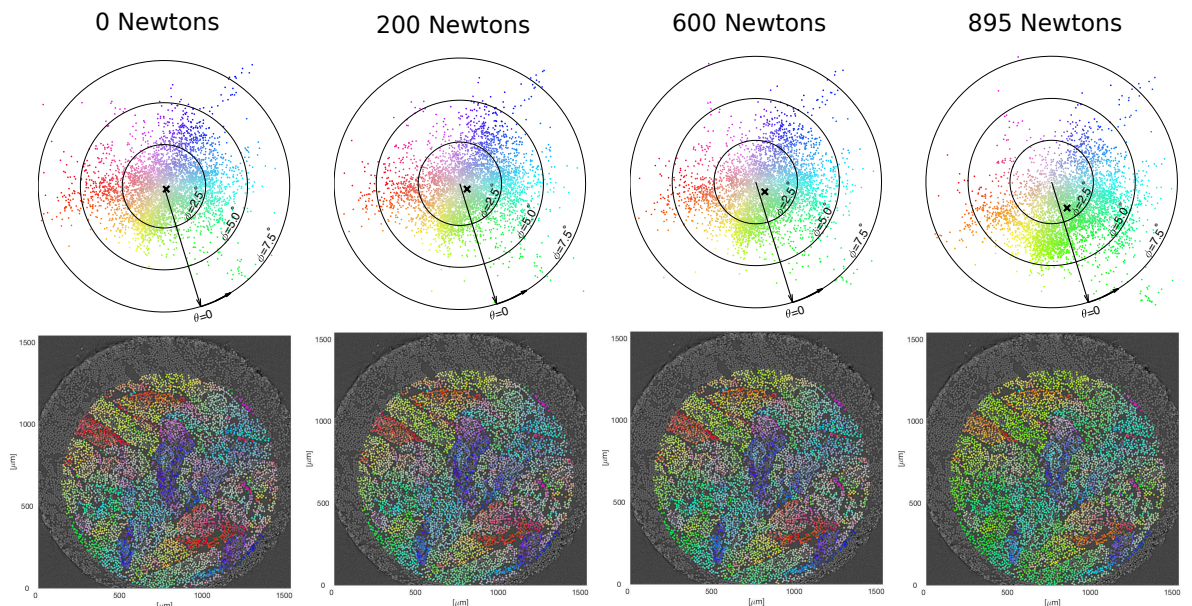

Figure 7: Spatial clustering of fibre orientations under load. The top row shows scatter plots of the fibre vectors $\mathbf{v}_{\mathrm{f}_{n}}^{i}$ for the four considered loading steps. Each point corresponds to a certain fibre $\mathrm{f}_{n}$ and is coloured according to: $\mathrm{i}$ ) inclination $\phi$ (colour saturation) and ii) azimuth $\theta$ (colour hue) of the fibre vector. The black crosses indicate the composite orientation $\mathbf{v}_{\mathrm{c}}^{i}$ (average over all fibres). In the bottom row the X-ray CT cross-section for the middle slice of the RoI is shown with the fibres displayed using the same colour as in the top row, but at their actual spatial location in the material.

unloaded sample where all deflections are zero. They show that even at the $200 \mathrm{~N}$ most of the fibres have already deflected towards the direction defined by the kink-band plane, although the magnitude of the deflection is still small.

The deflection vectors in these scatter plots have been colour-coded relative to the composite deflection $\mathbf{d}_{\mathrm{c}}^{i}$, which is indicated with a black cross over each scatter plot. The colours therefore indicate the inclination and the azimuth of the relative deflection vectors $\mathbf{r}_{\mathrm{f}_{n}^{i}}$ (recall that these capture the deflection of individual fibres with respect to the composite deflection). Thus, the colours in the cross-sectional slices in the bottom of Fig. 8 illustrate how the fibres in the different parts of the composite deflect relative to the mean. By looking at the spatial distribution of the relative deflection vectors it can be concluded that the deflection behaviour is not influenced by the initial orientation, as the clustering is not similar to that of the fibre vectors (see Fig. 7). Instead, all the fibres in the composite rod primarily undergo a cooperative deflection, as 
indicated by the black crosses over the scatter plots in Fig. 8 and a twist in an anti-clockwise direction with respect to the centre of the rod, indicated by the yellow arrows over the cross-sectional slices in Fig. 8.

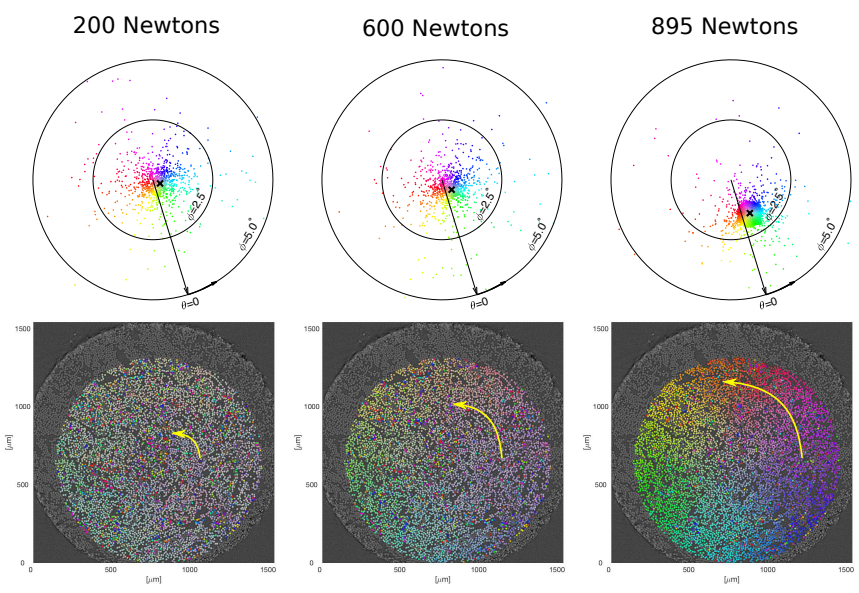

Figure 8: Evolution of deflection vectors and spatial distribution of relative deflection vectors under load. In the top row the scatter plots of the deflection vectors $\mathbf{d}_{f_{n}}$. The colour-coding has been set relative to the deflection of the composite rod, indicated by the black crosses. Thus, when shown at their actual location in the bottom row, the colours show the orientation of the relative deflection vectors $\mathbf{r}_{\mathrm{f}_{n}}$. The yellow arrows indicate the twist observed around the centre of the sample, note that the length of the arrows does not represent the absolute magnitude of the twist, which is $1.06^{\circ}$ at $895 \mathrm{~N}$.

\subsection{Evolution of composite deflection under load}

The histograms for the inclination and azimuth of the fibre vectors are reported for all loads in Fig. 9(a-b). Note in Fig. 9(a) that the spread of fibre inclinations increases somewhat as the load increases, and that fibres are tilting. The direction in which the fibres are tilting is reported in Fig. 9(b). There, it can be seen that fibres are not uniformly distributed around the full $360^{\circ}$ circle. Preferential directions exist and it is noticeable that there is a deficit of fibres at $-180 / 180^{\circ}$ to the kink-band direction $\left(0^{\circ}\right)$. This might be the reason for the kink band forming in this specific direction, however it could also be due to the freedom of movement in the $x y$ plane, due to end-loading the sample without 
shown as a function of loading in terms of i) the composite deflection (inclination of the composite segment in the FoV), in Fig. 9(c), and ii) the twist of the composite rod about its central axis, in Fig. 9(d). Unsurprisingly, the angle of inclination of the rod segment moves off axis faster as the load increases, but of the final kink band (see the scatter plots in Fig. 8), already at $25 \%$ of the kink-band collapse load.

The twist angle increases with axial compression load, and amounts to $1.06^{\circ}$ just prior to failure. On the one hand, the twist could arise from non-ideal loading, which could introduce some torque onto the specimen. While a rotational moment was not intentionally applied, it is possible that imprecise alignment of top and bottom jaws introduced some degree of rotational movement. On the other hand, the twist could have already been inside the unloaded sample. Regarding the effect of this twist on the damage evolution, it is expected that the twist will result in some degree of fibre deflection out of the dominant kink-band plane, such that the collapse mechanism has a 3D nature.

\section{Conclusions}

In this study the $3 \mathrm{D}$ orientations of individual fibres under load have been measured and tracked experimentally for the first time by means of time-lapse Xthe changes in fibre orientation to a very high level of accuracy, tracking the great majority of fibres in the composite. This approach has allowed to better visualise and understand precursors to fibre micro-buckling in a GFRP under compression loading. The main findings are considered below.

330

It is a good question to ask whether the ultimate kink-band location and failure direction is predestined from the start. This paper reports only the average orientations of individual fibres along the RoI and does not set out to measure the change in the curvature of the fibres. This will be the subject of 


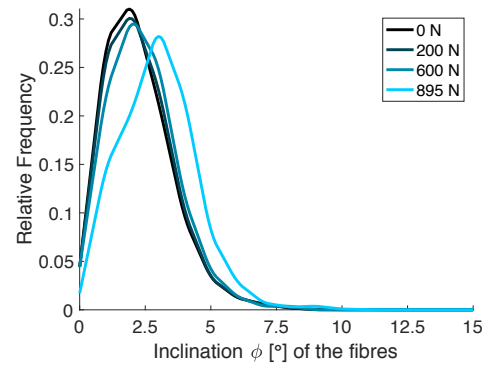

(a)

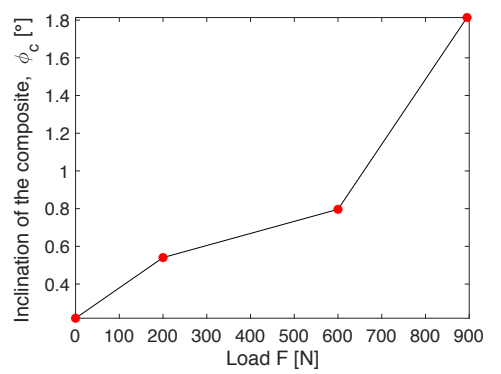

(c)

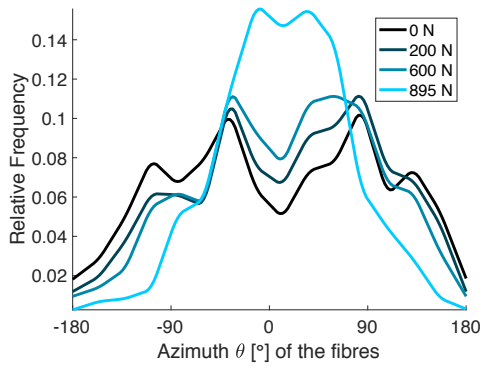

(b)

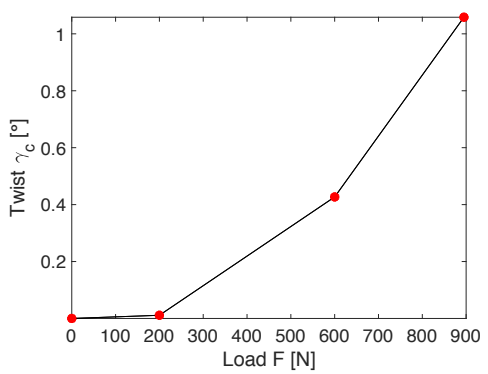

(d)

Figure 9: Progression of histograms capturing the magnitude and direction of fibre misalignment for the fibre vectors and the trend of the composite behaviour under load. In (a) and (b) the histograms for the inclination and azimuth of the fibre vectors. In (c) and (d) the trends for the composite tilt and twist.

a further paper. It is clear from Fig. 6 that the gauge section contains a number of misaligned clusters of around $200 \mu \mathrm{m}$ in diameter. It is worthwhile to consider whether the gauge is large enough to be statistically representative of the composite as a whole. It seems that the test piece contains around $30-40$ clusters and so it is probably just large enough for it to be unlikely that a particular cluster would dominate the compressive failure behaviour. Nevertheless, it is useful to ask whether the average misalignment angle/direction of all the fibres affects the initiation of the micro-buckling behaviour. Certainly it was remarked upon earlier that there appears to be few fibres orientated at $180^{\circ}$ to the kink direction (Fig. 9(b)) and this may help explain why the fibre tilting and eventual micro-buckling occurs in the direction it does. When considering the relative deflection vectors in Fig. 8, we can infer that there is no tendency 
for longitudinal splitting between fibres (no neighbouring clusters move in opposite directions laterally, as illustrated in Fig. 1(d)). Without information about the local curvature we are not able to identify whether the fibres buckle solely as depicted in Fig. 1 (c) or whether there is an increase in the local curvatures as in Fig. 1 (b). Certainly our work shows for the first time that the deflection initiates at very low loads and is in a direction close to the ultimate kink-band direction. Furthermore, this deflection increase accelerates as load increases such that the composite segment in the RoI is inclined by $1.81^{\circ}$ at $895 \mathrm{~N}$ (just prior to buckling instability). It is also clear that despite aiming to impose solely axial compression, there is a tendency for the composite to twist under increasing load, introducing another loading mode onto the composite. If the shear modulus of the composite is around $3 \mathrm{GPa}$ (adapted from Hull and Clyne [35]), the $1.06^{\circ}$ of twist equates to a torque of around $0.02 \mathrm{Nm}$. Ultimately, the notch does ensure that the final failure sequence occurs local to it, but the current analysis method does not pick up any local reorientation of the fibres in the periphery of the gauge volume associated with the notch because it approximates each measured fibre trajectory with a single straight line. Of course future measurements of the fibre curvature may provide indication of notch localised fibre curvature in the gauge volume. Finally, it should be remarked that the methodology presented in this paper, which can follow changes in individual fibres under load, is applicable to a wide range of materials based on UD fibres, including carbon fibre reinforced polymers.

\section{Acknowledgements}

The authors would like to gratefully acknowledge Dr. Rajmund Mokso, former beamline scientist at TOMCAT (SLS), and Dr. Eric Maire at INSALyon MATEIS. Financial support from CINEMA (the alliance for imaging of energy materials) [DSF-grant no. 1305-00032B] under the Danish Council for Strategic Research is acknowledged and P.J.W. is grateful to the European Research Council for the funding under CORREL-CT [grant no. 695638]. 
[1] C. Soutis, Carbon fiber reinforced plastics in aircraft construction, Materials Science and Engineering: A 412 (1) (2005) 171-176.

[2] P. Brøndsted, H. Lilholt, A. Lystrup, Composite materials for wind power turbine blades, Annu. Rev. Mater. Res. 35 (2005) 505-538.

380 [3] P. P. Camanho, S. R. Hallett, Numerical modelling of failure in advanced composite materials, Woodhead Publishing, 2015.

[4] G. D. Sims, Review of composites standardisation status and activities, National Composites Network, UK (2007).

[5] B. Budiansky, N. A. Fleck, Compressive failure of fibre composites, Journal of the Mechanics and Physics of Solids 41 (1) (1993) 183-211.

[6] N. Fleck, Compresssive failure of fiber composites, Advances in Applied Mechanics 33 (1997) 43-118.

[7] C. R. Schultheisz, A. M. Waas, Compressive failure of composites, parts i and ii, Progress in Aerospace Sciences 32 (1) (1996) 1-78.

[8] K. Niu, R. Talreja, Modeling of compressive failure in fiber reinforced composites, International Journal of Solids and Structures 37 (17) (2000) 24052428.

[9] I. Guz, M. Menshykova, C. Soutis, Internal instability as a possible failure mechanism for layered composites, The Philosophical Transactions of the Royal Society A 374 (2071) (2016) 20160019.

[10] N. F. Dow, I. J. Gruntfest, Determination of most needed potentially possible improvements in materials for ballistic and space vehicles, General Electric Company, Air Force Contract AF 4 (647) (1960) 269.

[11] C. Soutis, Compressive strength of unidirectional composites: measurement and prediction, in: Composite Materials: Testing and Design, Thirteenth Volume, ASTM International, 1997. 
[12] M. R. Wisnom, J. Atkinson, Fibre waviness generation and measurement and its effect on compressive strength, Journal of Reinforced Plastics and Composites 19 (2) (2000) 96-110.

[13] S. Pimenta, R. Gutkin, S. Pinho, P. Robinson, A micromechanical model for kink-band formation: Part iexperimental study and numerical modelling, Composites Science and Technology 69 (7) (2009) 948-955.

[14] E. G. Guynn, W. L. Bradley, O. O. Ochoa, A parametric study of variables that affect fiber microbuckling initiation in composite laminates: Part 1analyses, part 2experiments, Journal of Composite Materials 26 (11) (1992) 1594-1643.

[15] P. Moran, X. Liu, C. Shih, Kink band formation and band broadening in fiber composites under compressive loading, Acta Metallurgica et Materialia 43 (8) (1995) 2943-2958.

[16] S. Garcea, Y. Wang, P. Withers, X-ray computed tomography of polymer composites, Composites Science and Technology 156 (2018) 305-319.

[17] Y. Wang, T. L. Burnett, Y. Chai, C. Soutis, P. J. Hogg, P. J. Withers, X-ray computed tomography study of kink bands in unidirectional composites, Composite Structures 160 (2017) 917-924.

[18] Y. Wang, S. Garcea, P. Withers, 7.6 Computed Tomography of Composites, in: Comprehensive Composite Materials II, Ref. Module Mater. Sci. Mater. Eng., Elsevier, Oxford, 2018, pp. 101-118.

[19] Y. Wang, S. C. Garcea, T. Lowe, E. Maire, C. Soutis, P. J. Withers, Ultrafast time-lapse synchrotron radiographic imaging of compressive failure in CFRP, in: 17th European Conference on Composite Materials, Munich, Germany, 2016.

[20] S. C. Garcea, I. Sinclair, S. M. Spearing, P. J. Withers, Mapping fibre failure in situ in carbon fibre reinforced polymers by fast synchrotron $\mathrm{X}$ - 
ray computed tomography, Composites Science and Technology 149 (2017) 81-89.

[21] Y. Wang, Damage mechanisms associated with kink-band formation in unidirectional fibre composites, Ph.D. thesis, University of Manchester (2016).

[22] H. Altendorf, D. Jeulin, 3D directional mathematical morphology for analysis of fiber orientations, Image Analysis \& Stereology 28 (3) (2011) 143-153.

[23] M. Tausif, B. Duffy, S. Grishanov, H. Carr, S. J. Russell, Three-dimensional fiber segment orientation distribution using X-ray microtomography, Microscopy and Microanalysis 20 (4) (2014) 1294-1303.

[24] O. Wirjadi, K. Schladitz, P. Easwaran, J. Ohser, Estimating fibre direction distributions of reinforced composites from tomographic images, Image Analysis \& Stereology 35 (3) (2016) 167-179.

[25] G. Requena, G. Fiedler, B. Seiser, P. Degischer, M. Di Michiel, T. Buslaps, 3D-quantification of the distribution of continuous fibres in unidirectionally reinforced composites, Composites Part A: Applied Science and Manufacturing 40 (2) (2009) 152-163.

[26] M. W. Czabaj, M. L. Riccio, W. W. Whitacre, Numerical reconstruction of graphite/epoxy composite microstructure based on sub-micron resolution X-ray computed tomography, Composites Science and Technology 105 (2014) 174-182.

[27] R. Sencu, Z. Yang, Y. Wang, P. Withers, C. Rau, A. Parson, C. Soutis, Generation of micro-scale finite element models from synchrotron X-ray CT images for multidirectional carbon fibre reinforced composites, Composites Part A: Applied Science and Manufacturing 91 (2016) 85-95.

[28] M. J. Emerson, K. M. Jespersen, A. B. Dahl, K. Conradsen, L. P. Mikkelsen, Individual fibre segmentation from 3D X-ray computed tomography for characterising the fibre orientation in unidirectional com- 
posite materials, Composites Part A: Applied Science and Manufacturing 97 (2017) 83-92.

[29] M. J. Emerson, V. A. Dahl, K. Conradsen, L. P. Mikkelsen, A. B. Dahl, Statistical validation of individual fibre segmentation from tomograms and microscopy, Composites Science and Technology 160 (2018) 208-215.

[30] D. Paganin, S. Mayo, T. E. Gureyev, P. R. Miller, S. W. Wilkins, Simultaneous phase and amplitude extraction from a single defocused image of a homogeneous object, Journal of Microscopy 206 (1) (2002) 33-40.

[31] E. Maire, C. Le Bourlot, J. Adrien, A. Mortensen, R. Mokso, 20 Hz X-ray tomography during an in situ tensile test, International Journal of Fracture 200 (1-2) (2016) 3-12.

[32] V. A. Dahl, C. H. Trinderup, M. J. Emerson, A. B. Dahl, Content-based propagation of user markings for interactive segmentation of patterned images, ArXiv e-printsarXiv: 1809.02226.

${ }_{470}$ [33] M. Ueda, K. Mimura, T.-K. Jeong, In situ observation of kink-band formation in a unidirectional carbon fiber reinforced plastic by X-ray computed tomography imaging, Advanced Composite Materials 25 (1) (2016) 31-43.

[34] K. S. Arun, T. S. Huang, S. D. Blostein, Least-squares fitting of two 3-d point sets, IEEE Trans. Pattern Anal. Mach. Intell. (5) (1987) 698-700.

475 [35] D. Hull, T. Clyne, An introduction to composite materials, Cambridge university press, 1996. 